\title{
Multimodal multiphoton imaging for label-free monitoring of early gastric cancer
}

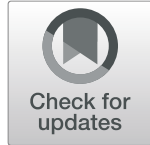

Lianhuang $\mathrm{Li}^{{ }^{*+}}$, Deyong Kang ${ }^{2 \dagger}$, Zicheng Huang ${ }^{3 \dagger}$, Zhenlin Zhan ${ }^{1}$, Changyin Feng ${ }^{2}$, Yongjian Zhou ${ }^{4^{*}}$, Haohua Tu ${ }^{5^{*}}$, Shuangmu Zhuo ${ }^{1}$ and Jianxin Chen ${ }^{1}$

\begin{abstract}
Background: Early gastric cancer is associated with a much better prognosis than advanced disease, and strategies to improve prognosis is strictly dependent on earlier detection and accurate diagnosis. Therefore, a label-free, non-invasive imaging technique that allows the precise identification of morphologic changes in early gastric cancer would be of considerable clinical interest.

Methods: In this study, multiphoton microscopy (MPM) using two-photon excited fluorescence combined with second-harmonic generation was used for the identification of early gastric cancer.

Results: This microscope was able to directly reveal improved cellular detail and stromal changes during the development of early gastric cancer. Furthermore, two features were quantified from MPM images to assess the cell change in size and stromal collagen change as gastric lesion developed from normal to early cancer.

Conclusions: These results clearly show that multiphoton microscopy can be used to examine early gastric cancer at the cellular level without the need for exogenous contrast agents. This study would be helpful for early diagnosis and treatment of gastric cancer, and may provide the groundwork for further exploration into the application of multiphoton microscopy in clinical practice.
\end{abstract}

Keywords: Early gastric cancer, Multiphoton imaging, Two-photon excited fluorescence, Second-harmonic generation

\section{Background}

Early gastric cancer is defined as gastric cancer that is confined to the mucosa or submucosa, regardless of the presence or absence of regional lymph node metastasis $[1$, 2]. The prognosis for early gastric cancer is universally excellent, and strategies to improve prognosis is strictly dependent on earlier detection and accurate diagnosis since early gastric cancer can potentially be cured by endoscopic therapy such as endoscopic mucosal resection

\footnotetext{
* Correspondence: Ihli@fjnu.edu.cn; zhouyjbju@163.com; htu@illinois.edu ${ }^{\dagger}$ Lianhuang Li, Deyong Kang and Zicheng Huang these authors contributed equally to this work.

${ }^{1}$ Key Laboratory of OptoElectronic Science and Technology for Medicine of Ministry of Education, Fujian Provincial Key Laboratory for Photonics Technology, Fujian Normal University, Fuzhou 350007, People's Republic of China

${ }^{4}$ Department of Gastric Surgery, Fujian Medical University Union Hospital, Fuzhou 350001, People's Republic of China

${ }^{5}$ Beckman Institute for Advanced Science and Technology, University of Illinois at Urbana-Champaign, Urbana, IL 61801, USA

Full list of author information is available at the end of the article
}

or endoscopic submucosal dissection [3, 4]. It was reported that patients with early gastric cancer after treatment had a 1 year survival in excess of 90\% [5]. However, there is little information on the symptoms of early gastric cancer and it is often difficult to detect gastric superficial lesions using conventional endoscopy with white-light imaging [6]. At present, the diagnosis of these lesions is always based on the pathologic assessment of endoscopic biopsy specimens. As such, superficially taken biopsies and sampling error for histopathology are common events, making it difficult to draw firm conclusions [7]. Hence, development of a new diagnostic technique will be of important clinical meaning.

Multiphoton imaging technique such as two-photon excitation fluorescence (TPEF) and second-harmonic generation (SHG) has been widely used for biological tissue imaging $[8,9]$. TPEF exploits the auto-fluorescence of biological samples and therefore obviates the need for exogenous contrast agents, and SHG makes use of the

(C) The Author(s). 2019 Open Access This article is distributed under the terms of the Creative Commons Attribution 4.0 International License (http://creativecommons.org/licenses/by/4.0/), which permits unrestricted use, distribution, and 
non-centrosymmetric properties to image structural proteins and will not suffer from phototoxicity effects or photobleaching because it does not involve excitation of molecules [10-12]. There are a variety of intracellular molecules including $\mathrm{NADH}, \mathrm{FAD}$ and porphyrins, as well as certain extracellular components such as elastin and collagen within gastric tissues, which can generate intrinsic multiphoton signals. Therefore, in this study, we investigated the potential of using label-free, multimodal multiphoton microscopy that incorporates two-photon excited fluorescence and second-harmonic generation techniques for distinguishing early gastric cancer from normal tissues.

\section{Methods}

\section{Imaging instrumentation}

As has been described previously, a mode-locked femtosecond Ti:sapphire laser (Mira 900-F, Coherent, Inc., USA) was used as the multiphoton excitation source, and the excitation light was delivered to and the emitted light was also collected from the sample through an inverted microscope (LSM 510 META, Zeiss, Germany) $[13,14]$. A $63 \times$ Zeiss Plan-Apochromat oil immersion objective lens (Numerical Aperture (NA) =1.4) was chosen for capturing multiphoton microscopic images. The backscattered intrinsic TPEF and SHG signals were received respectively by a META detector composed of a reflective grating and an optimized 32-channel PMT array detector using $810 \mathrm{~nm}$ excitation wavelength. TPEF signal was detected in the wavelength range 430-716 nm and SHG signal was detected in the 389 to $419 \mathrm{~nm}$ wavelength range. In order to provide a visual contrast of multiphoton images, all the TPEF images adopted pseudo-colored red and SHG images were marked with pseudo-colored green.

\section{Sample preparation}

In this work, 12 early gastric cancer samples including 1 well-differentiated adenocarcinoma, 6 moderately-differentiated adenocarcinomas, 1 moderately-poorly differentiated adenocarcinoma and 4 poorly-differentiated adenocarcinomas were collected. All samples were immediately sent to the pathology laboratory for frozen section through a cryostat microtome once they were obtained from the surgeons. Three consecutive sections with $10 \mu \mathrm{m}$ thickness were used in this study, in which two slices were used to carry out the multiphoton microscopic imaging and the middle section was diagnosed through hematoxylin and eosin staining $(H \& E)$ in order to further determine the experimental results. In addition, we also collected 12 normal gastric tissue samples for the sake of comparison. One thing to be aware of was that in order to avoid specimen shrinkage or dehydration, we added a small amount of phosphate-buffered saline (PBS) to the tissue sections during the experiment.

\section{Histologic analysis}

Firstly, every H\&E-stained slice was checked by two certified pathologists in this study to avoid the inter-individual variability, and then a digital image of the H\&E-stained slide was acquired by an optical microscope (Eclipse Ci-L, Nikon Instruments Inc., Japan) with a CCD (DS-Fi2, Nikon). Secondly, two investigators who were blinded to the diagnostic results confirmed all the multiphoton imaging results by comparing with the H\&E-stained digital images.

\section{Statistical analysis}

Furthermore, in the development of early gastric cancer, we measured the circumference of cell nucleus and SHG average intensity per pixel so that the changes of cell size and collagen content in mucosa could be quantitatively estimated. For every multiphoton image, SHG average intensity per pixel was obtained via dividing the sum of all intensities by the total number of pixels [15]. The results were presented as a mean followed with its standard deviation (mean $\pm \mathrm{SD}$ ). In this study, we made use of the ImageJ software for analyzing SHG images, and based on the IBM SPSS Statistics 21, we also employed the student's t-test to carry out statistical analysis, and $P$ value less than 0.05 was regarded as statistical significance.

\section{Results MPM imaging}

The focus of the first part of this work is to qualitatively determine whether MPM can effectively identify the morphologic differences between normal and early cancerous gastric tissues. Figure 1 shows representative MPM images of normal gastric mucosa and a corresponding H\&E-stained image. MPM images reveal that mucosal tissues mainly consist of gastric glands and collagen fibers. Specifically, an individual epithelial cell nucleus (white arrow in Fig. 1b) which is located at basement membrane surface could be identified, and individual gastric gland composed of epithelial cells is readily differentiated via TPEF image. Normal cells are uniform in shape with small nuclei, and the gastric glands arrange regularly with uniform shape. These same details of cellular and subcellular architecture correlate well with the corresponding H\&E-stained image (Fig. 1d). It is well-known that collagen matrix forms the basic framework of stroma in mucosa, and plays an important role in the development of cancer [16]. SHG image displays that the basement membrane (yellow arrow in Fig. 1a) is identified readily as a thin band surrounding individual gland, and collagen fibers in stroma form a fine reticular structure for sustaining gastric 

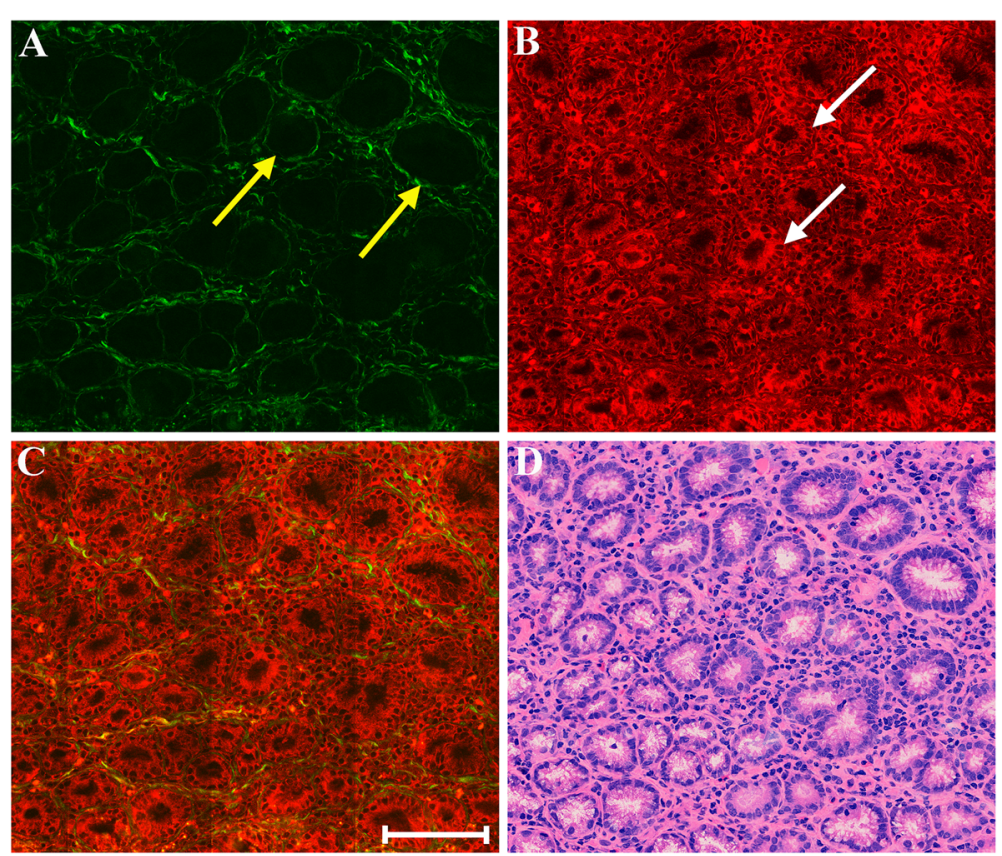

Fig. 1 MPM images of normal gastric mucosa and corresponding H\&E-stained image. a SHG image; b TPEF image; c Merging of SHG and TPEF images; d H\&E-stained image. Yellow arrow: basement membrane; white arrow: epithelial cells. Scale bar: 100 um

glands. Compared with the H\&E-stained image, the extracellular matrix is more prominent in SHG image.

Early gastric cancer can be divided into those that invade the mucosa only, and those that penetrate the muscularis mucosa to the submucosa. Figure 2 shows representative MPM images of early gastric cancer that has invaded mucosa alone and a corresponding H\&E-stained image. TPEF image demonstrates that the morphology of individual nuclei is observed readily, and the intercellular space between individual cells could be discerned as well. Compared with normal tissues, cancerous cell nuclei grow in size significantly and cellular pleomorphism is obvious, and the gastric glands are disordered and are of various shapes.

More importantly, abnormal cells have broken through the basement membrane and invaded into the stroma sparsely and diffusely (white arrow in Fig. 2b), which makes it impossible to detect by other optical imaging techniques [17]. These features are comparable with what is obtained using standard H\&E histology (Fig. 2d). SHG image displays that connective tissues which are mainly made up of collagen fibers and are used for
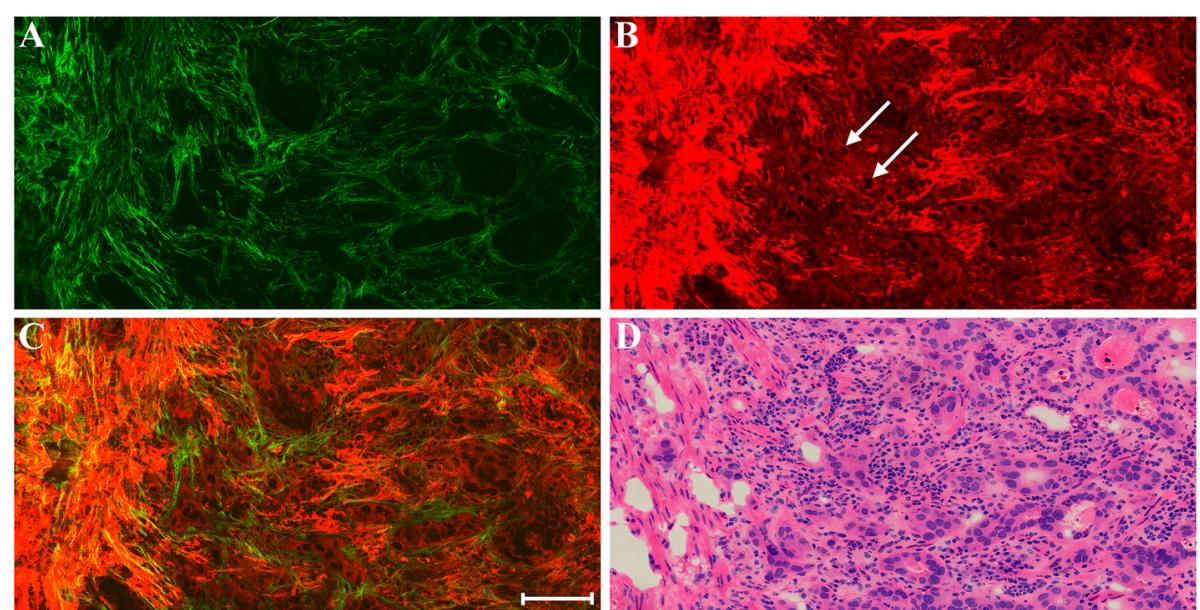

Fig. 2 MPM images of early gastric cancer that has invaded mucosa alone and corresponding H\&E-stained image. a SHG image; b TPEF image; c Merging of SHG and TPEF images; d H\&E-stained image. White arrow: cancerous cells. Scale bar: $100 \mu \mathrm{m}$ 
separating individual glands could be detected too, but they are chaotic and gather together in comparison with normal gastric mucosa. The change of collagen fibers is therefore considered to reflect the development of gastric cancer.

Figure 3 shows representative MPM images of early gastric cancer that has invaded into submucosa and corresponding H\&E-stained images. It can be clearly seen that malignant tumors have penetrated the muscularis mucosae (yellow arrow in Fig. 3c) to the submucosa. MPM images also reveal that the submucosal layer mainly consists of collagen fibers and elastic fibers. Thus it is easy to find tumor invasion (blue arrow in Fig. 3e) by MPM examination. Additionally, blood vessel (pink arrow in Fig. 3e) can be identified readily as elastin in the vascular wall is an endogenous fluorescent molecule, and can emit TPEF signal. These qualitative morphological variations correspond to the corresponding H\&E-stained image of paired histological section.
Figure 4 shows representative MPM images of a boundary between submucosal and muscular layers in early gastric cancer and a corresponding H\&E-stained image. Morphologically, malignant tumors (white arrow in Fig. 4b) have invaded into the boundary between submucosal and muscular layers, but were still confined to the submucosa. These morphologic changes are consistent with those identified with the corresponding H\&E-stained image (Fig. 4d). Unlike in normal tissues, collagen fibers in submucosa are disrupted by tumor invasion and even almost disappear. The muscularis propria (blue arrow in Fig. 4c) could be detected too by MPM image. The definition of early gastric cancer allows for invasion only as far as the submucosa, without penetration through the muscularis propria. Although tumors did not invaded into the muscular layer, elastic fibers (yellow arrow in Fig. 4b) have been affected such as fracture, aggregation. Together, these findings suggest that MPM may be useful for identifying not only normal gastric tissues but also malignant gastric lesions.
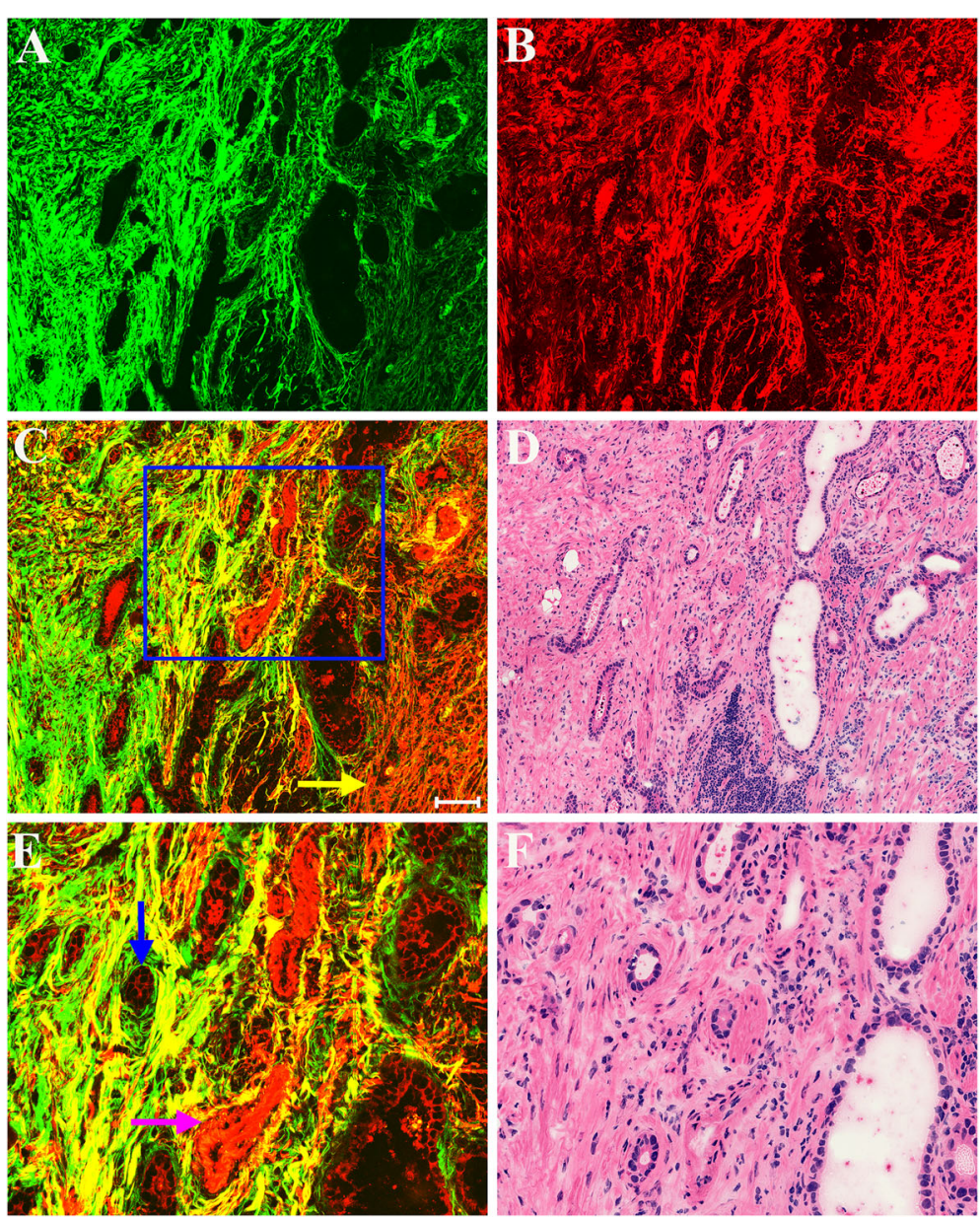

Fig. 3 MPM images of early gastric cancer that has invaded into submucosa and corresponding H\&E-stained images. a SHG image; b TPEF image; $\mathbf{c}$ Merging of SHG and TPEF images; $\mathbf{d}$ H\&E-stained image; and e-f Zoom-in image of the boxed region in (c) and corresponding H\&Estained image. Yellow arrow: muscularis mucosae; blue arrow: tumor cells; pink arrow: blood vessel. Scale bar: $100 \mu \mathrm{m}$ 

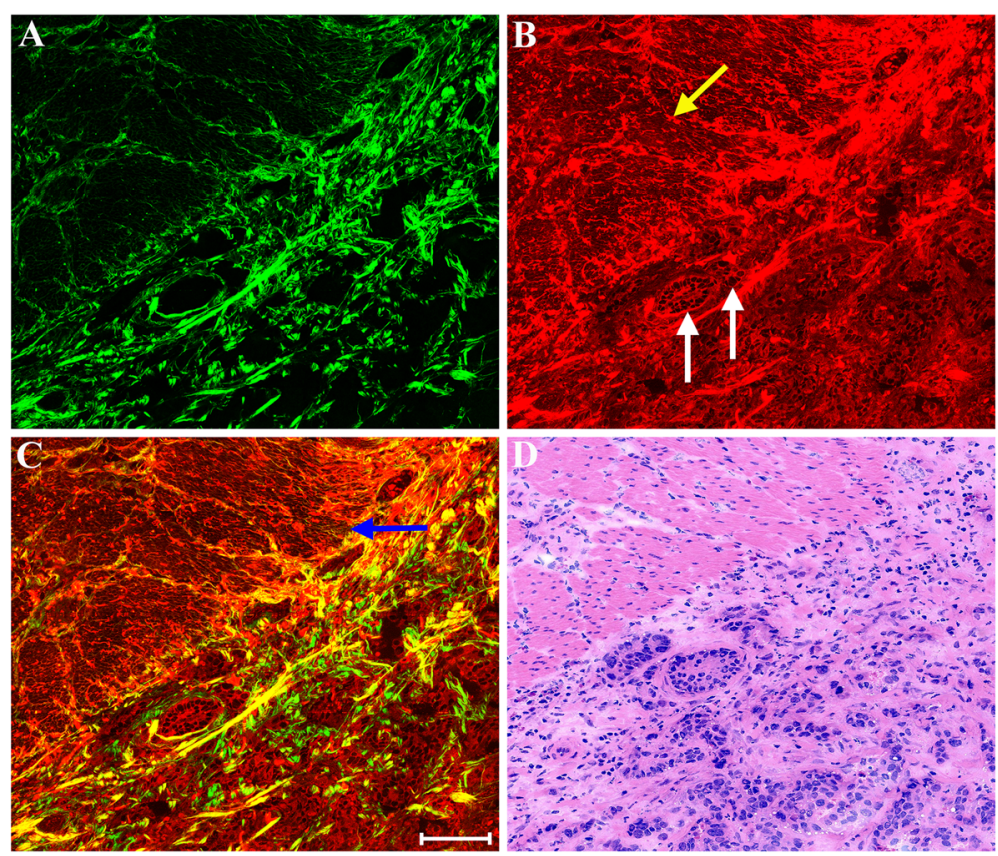

Fig. 4 MPM images of a boundary between submucosal and muscular layers in early gastric cancer and corresponding H\&E-stained image. a SHG image; $\mathbf{b}$ TPEF image; $\mathbf{c}$ Merging of SHG and TPEF images; $\mathbf{d}$ H\&E-stained image. Yellow arrow: elastic fiber; white arrow: malignant tumors; blue arrow: muscularis propria. Scale bar: $100 \mu \mathrm{m}$

\section{Quantitative analysis}

To determine whether these morphologic features were statistically different between normal and early gastric cancer tissues, two features in mucosa were quantified from MPM images. The nucleus circumference was measured for assessing the change in cell size, and SHG average intensity per pixel was calculated to quantify stromal collagen change. As shown in Table 1, the average and SD of normal cell nucleus circumference is $20.32 \pm 2.38 \mu \mathrm{m}$, and of cancerous cell nucleus circumference is $47.25 \pm 6.41 \mu \mathrm{m}$, indicating that the cell nucleus is significantly enlarged when gastric disease progresses from normal to early cancer. There is a statistically highly significant difference $(P<0.002)$ between normal and early gastric cancer.

It has become evident that collagen matrix plays an important role in the progression of early gastric cancer. Quantitative results shown in Table 1 reveal that the collagen fibers in mucosa tend to increase as gastric disease develops from normal to early cancer. More specifically,

Table 1 Quantitative variables for distinguishing normal and early gastric cancer tissues with multiphoton microscopy

\begin{tabular}{|c|c|c|}
\hline \multirow[t]{2}{*}{ Samples } & \multicolumn{2}{|l|}{ Quantitative variables } \\
\hline & $\begin{array}{l}\text { Nucleus circumference } \\
(\mu \mathrm{m})\end{array}$ & $\begin{array}{l}\text { SHG average intensity } \\
\text { per pixel }\end{array}$ \\
\hline Normal $(n=12)$ & $20.32 \pm 2.38$ & $16.76 \pm 1.26$ \\
\hline $\begin{array}{l}\text { Early gastric cancer } \\
(n=12)\end{array}$ & $47.25 \pm 6.41$ & $25.59 \pm 4.47$ \\
\hline
\end{tabular}

the SHG average intensity per pixel in normal mucosa is $16.76 \pm 1.26$, however, in cancerous mucosa is $25.59 \pm$ 4.47. Statistically significant difference $(P<0.03)$ in the SHG average intensity per pixel was also found between normal and early gastric cancer. It is clear that the collagen content within gastric tissues is altered with the progression of early cancer, and thus the detection of the signal from collagen could provide us an approach to differentiate normal from abnormal tissues.

\section{Assessment of diagnostic accuracy}

Lastly, we performed a blinded study to evaluate the diagnosis precision by MPM based on the optical diagnostic features. Twenty-four samples, including 12 normal cases and 12 abnormal cases were analyzed in this work. A doctor and a researcher were invited for this pilot study, and they were all blinded to the diagnosis information of the patients. The diagnosis results from the MPM and H\&E staining were shown in Table 2. More specifically, the diagnosis sensitivity was $91.7 \%$, the specificity was $100 \%$, the positive predictive value (PPV) was $100 \%$, and the negative predictive value (NPV) was 92.3\%. The false-negative rate (FNR) was $8.3 \%$ because one early gastric cancer was diagnosed as normal case according to MPM examination. It is important to point out that the test accuracy may be limited because of limited samples and therefore further studies will need to be performed to verify these findings. 
Table 2 Diagnosis results from the multiphoton microscopy and final histopathologic diagnosis respectively

\begin{tabular}{|c|c|c|c|c|}
\hline & & \multicolumn{2}{|l|}{ MPM Diagnosis } & \\
\hline & & Normal & Cancer & \\
\hline \multirow[t]{3}{*}{ H\&E Diagnosis } & Normal & 12 & 0 & $P P V=100 \%$ \\
\hline & Cancer & 1 & 11 & $N P V=92.3 \%$ \\
\hline & & Sensitivity $=91.7 \%$ & Specificity $=100 \%$ & \\
\hline
\end{tabular}

Abbreviations: MPM multiphoton microscopy, H\&E hematoxylin-eosin, PPV positive predictive value, NPV negative predictive value

\section{Discussion}

Most gastric cancer is diagnosed at an advanced stage with a poor survival rate, despite treatment including major surgery and adjuvant therapy. The outcome could be improved by early detection and treatment $[18,19]$. Because early gastric cancer is associated with a much better prognosis than advanced disease, its diagnosis is important. Endoscopic screening is widely accepted as an available approach for early gastric cancer because it is minimally invasive, safe and convenient. However, conventional endoscopy examination has limited ability to precisely identify this lesion because of the insufficient resolution [20]. The disadvantages existing in MRT (Magnetic Resonance Imaging), CT (Computed Tomography), US (Ultrasound), and so on should not be overlooked, such as low resolution, poor contrast sensitivity, or even potential radioactive hazard [17]. Histological evaluation of biopsy specimens is still the golden standard for diagnosing early gastric cancer, but performing biopsy procedures has several shortcomings, including sampling error, costs, risks to the patient, and the delay in obtaining results [21]. All these factors together might lead to underdiagnosis or overdiagnosis and therefore suboptimal treatment as well as surveillance practices.

The advantages presented by multiphoton microscopy such as not only the low photodamage and photobleaching, high-resolution, high-sensitivity as well as high-contrast, but also providing label-free detection of biological tissues in real time, may help reduce sampling errors and even eliminate the need for invasive tissue removal [22]. TPEF from NADH as well as FAD in cells and SHG from collagen have been shown to be two major intrinsic signals for gastric mucosal tissues. MPM images show that normal gastric mucosa is mainly composed of well-aligned glands and well-organized extracellular collagen network that is essential for the maintenance of gastric glands, while abnormal mucosal tissues have disordered glands with various shapes and have chaotic extracellular collagen matrix. Stromal changes will be involved in neoplastic process for providing a tumor-promoting environment for cancer progression and metastasis $[8,23]$. The presence of collagen fibers is therefore considered to influence the development of gastric cancer. As a major component of extracellular matrix, the architectural properties of collagen fibers can be obtained by SHG imaging to reflect stromal changes in abnormal tissues. Furthermore, quantitative analysis proves that the cells will be enlarged with the progression of early gastric cancer and collagen content increases because of desmoplastic response which is characterized by dense collagen growth. A pilot study showed that the diagnosis results based on MPM have high accuracy, and the sensitivity, specificity, PPV, and NPV were 91.7\%, 100\%, $100 \%$, and $92.3 \%$ respectively.

Though it has been shown that collagen changes would participate in the development of cancerization [24, 25], however, interestingly enough, some researchers reported that malignant cells may secrete extracellular enzymes to degrade the collagen fibers at the invasion front $[8,26$, 27], while some works demonstrated that tumor invasion may activate fibroblasts and cause desmoplasia, and therefore increase deposition of extracellular collagen [28, 29]. In this study, our data showed that collagen density increases during gastric carcinoma progression, which is consistent with the previous results [13].

The submucosa mainly consists of loose connective tissue with blood vessels, and elastic fibers as well as collagen fibers are the major components of normal connective tissue. Elastin is a common fluorescence source, and this property makes TPEF imaging a convenient tool for illustrating the microstructures of elastin fiber and blood vessel. In normal submucosal tissues, elastin fibers have a long rope-like structure, and collagen fibers are well-organized. However, in abnormal tissues, elastin fibers have almost disappeared, and collagen fibers are sparse and disrupted because malignant cells would secrete extracellular enzymes to degrade the original tissue architecture and promote tumor progression into surrounding tissues [30].

Additionally, because of the insufficient resolution of conventional endoscopy, endoscopic surgery may fail to remove disseminated invasive cells that lie beyond the surgical resection border, which may lead to tumor recurrence and ultimately patient death. The data obtained in this work show that MPM is capable of detecting single abnormal cell and identifying stratified structure in gastric tissues, and therefore may be helpful for determining surgical margin as well as selecting an optimal treatment when it is successfully incorporated into endoscope in the near future. Although the light penetration of MPM is still limited at present, it has been improving 
with the development of a variety of technologies, such as a gradient index lens-based MPM or a compact and flexible MPM probe [31, 32]. It was reported that the penetration depth of MPM could reach millimeter-order [33]. Maybe the combination of gradient index lens and multiphoton probe is a best method to overcome the defect of image depth.

Furthermore, the exploration of multiphoton endoscope into intravital imaging that aims to translate this technique into the clinics has been performed; especially the design of compact and flexible MPM probes for future clinical applications of gastrointestinal tract has achieved significant advancement [21, 31, 34, 35]. With miniaturization and integration of this technology [36, 37], MPM could be incorporated into endoscopy equipment or robotic surgical systems [38-40], and thus can be used in imaging during live endoscopy to diagnose gastrointestinal diseases as well as would be helpful to determine the surgical margin accurately.

\section{Conclusions}

In summary, MPM is useful for visualizing mucosal and submucosal microstructure information with advantages over currently available endoscopy technologies, and has greater diagnostic performance for early gastric cancer. This study also shows that development of a multiphoton endomicroscope therefore has a practical meaning for performing virtual biopsies during routine endoscopic screening.

\section{Acknowledgements}

Not applicable.

\section{Funding}

The project was supported by the National Natural Science Foundation of China (Grant No. 81671730), the Joint Funds of Fujian Provincial Health and Education Research (Grant No. WKJ2016-2-28), the Special Funds of the Central Government Guiding Local Science and Technology Development (Grant No. 2017 L3009), the Natural Science Foundation of Fujian Province (Grant Nos. 2018 J07004, 2016 J05150), and the Program for Changjiang Scholars and Innovative Research Team in University (Grant No. IRT_15R10).

\section{Availability of data and materials}

The data analyzed during the current study are available from the corresponding author on reasonable request.

\section{Authors' contributions}

$L H L, Y J Z$ and JXC were responsible for conception and design. Data were obtained and analyzed by LHL, ZCH, ZLZ, CYF and DYK. SMZ and HHT provided technical support. All authors contributed to the analysis and interpretation of data, wrote, reviewed and approved the final manuscript.

\section{Authors' information}

Initials: Lianhuang Li (LHL), Deyong Kang (DYK), Zicheng Huang (ZCH), Zhenlin Zhan (ZLZ), Changyin Feng (CYF), Yongjian Zhou (YJZ), Shuangmu Zhuo (SMZ), Haohua Tu (HHT), Jianxin Chen (JXC).

\section{Ethics approval and consent to participate}

This study was conducted with the approval of the institutional review board at the Fujian Medical University Union Hospital and conformed with the institutional rules governing clinical investigations of human subjects in biomedical research, and prior to study participation, each patient signed an informed consent

\section{Consent for publication}

Not applicable.

\section{Competing interests}

The authors declare that they have no competing interests.

\section{Publisher's Note}

Springer Nature remains neutral with regard to jurisdictional claims in published maps and institutional affiliations.

\section{Author details}

${ }^{1}$ Key Laboratory of OptoElectronic Science and Technology for Medicine of Ministry of Education, Fujian Provincial Key Laboratory for Photonics Technology, Fujian Normal University, Fuzhou 350007, People's Republic of China. ${ }^{2}$ Department of Pathology, Fujian Medical University Union Hospital, Fuzhou 350001, People's Republic of China. ${ }^{3}$ Department of

Gastroenterology, The First Hospital of Quanzhou Affiliated to Fujian Medical University, Quanzhou 362002, People's Republic of China. ${ }^{4}$ Department of Gastric Surgery, Fujian Medical University Union Hospital, Fuzhou 350001, People's Republic of China. ${ }^{5}$ Beckman Institute for Advanced Science and Technology, University of Illinois at Urbana-Champaign, Urbana, IL 61801, USA.

Received: 4 May 2018 Accepted: 20 March 2019

Published online: 02 April 2019

\section{References}

1. Isomoto H, Shikuwa S, Yamaguchi N, Fukuda E, Ikeda K, Nishiyama H, Ohnita K, Mizuta Y, Shiozawa J, Kohno S. Endoscopic submucosal dissection for early gastric cancer: a large-scale feasibility study. Gut. 2009:58:331-6.

2. Ono H, Kondo H, Gotoda T, Shirao K, Yamaguchi H, Saito D, Hosokawa K, Shimoda T, Yoshida S. Endoscopic mucosal resection for treatment of early gastric cancer. Gut. 2001;48:225-9.

3. Soetikno R, Kaltenbach T, Yeh R, Gotoda T. Endoscopic mucosal resection for early cancers of the upper gastrointestinal tract. J Clin Oncol. 2005;23: $4490-8$.

4. Gotoda T, Yamamoto H, Soetikno RM. Endoscopic submucosal dissection of early gastric cancer. J Gastroenterol. 2006;41:929-42.

5. Everett SM, Axon AT. Early gastric cancer in Europe. Gut. 1997;41:142-50

6. Dohi O, Yagi N, Majima A, Horii Y, Kitaichi T, Onozawa Y, Suzuki K, Tomie A, Kimura-Tsuchiya R, Tsuji T, Yamada N, Bito N, Okayama T, Yoshida N, Kamada K, Katada K, Uchiyama K, Ishikawa T, Takagi T, Handa O, Konishi H, Naito Y, Yanagisawa A, Itoh Y. Diagnostic ability of magnifying endoscopy with blue laser imaging for early gastric cancer: a prospective study. Gastric Cancer. 2017:20:297-303.

7. Sanduleanu S, Driessen A, Gomez-Garcia E, Hameeteman W, de Bruine A, Masclee A. In vivo diagnosis and classification of colorectal neoplasia by chromoendoscopy-guided confocal laser endomicroscopy. Clin Gastroenterol Hepatol. 2010;8:371-8.

8. Xu X, Cheng J, Thrall MJ, Liu Z, Wang X, Wong ST. Multimodal non-linear optical imaging for label-free differentiation of lung cancerous lesions from normal and desmoplastic tissues. Biomed Opt Express. 2013;4:2855-68.

9. Mouras R, Bagnaninchi P, Downes A, Elfick A. Multimodal, label-free nonlinear optical imaging for applications in biology and biomedical science. J Raman Spectrosc. 2013;44:1373-8.

10. Zipfel WR, Williams RM, Christie R, Nikitin AY, Hyman BT, Webb WW. Live tissue intrinsic emission microscopy using multiphoton-excited native fluorescence and second harmonic generation. Proc Natl Acad Sci U S A. 2003; 100:7075-80

11. Yeh AT, Nassif N, Zoumi A, Tromberg BJ. Selective corneal imaging using combined second-harmonic generation and two-photon excited fluorescence. Opt Lett. 2002;27:2082-4.

12. Campagnola PJ, Loew LM. Second-harmonic imaging microscopy for visualizing biomolecular arrays in cells, tissues and organisms. Nat Biotechnol. 2003:21:1356-60.

13. Li LH, Chen ZF, Wang XF, Liu X, Jiang WZ, Zhuo SM, Jiang LW, Guan GX, Chen JX. Monitoring neoadjuvant therapy responses in rectal cancer using multimodal nonlinear optical microscopy. Oncotarget. 2017;8:107323-33. 
14. Chen JX, Xu J, Kang DY, XU MF, Zhuo SM, Zhu XQ, Jiang XS. Multiphoton microscopic imaging of histological sections without hematoxylin and eosin staining differentiates carcinoma in situ lesion from normal oesophagus. Appl Phys Lett. 2013;103:183701.

15. Birk JW, Tadros M, Moezardalan K, Nadyarnykh O, Forouhar F, Anderson J, Campagnola P. Second harmonic generation imaging distinguishes both high-grade dysplasia and Cancer from Normal colonic mucosa. Dig Dis Sci. 2014:59:1529-34.

16. Zheng W, Li D, Li S, Zeng Y, Yang Y, Qu JY. Diagnostic value of nonlinear optical signals from collagen matrix in the detection of epithelial precancer. Opt Lett. 2011:36:3620-2.

17. Weissleder R, Pittet MJ. Imaging in the era of molecular oncology. Nature. 2008:452:580-9.

18. Conio M, Ponchon T, Blanchi S, Filiberti R. Endoscopic mucosal resection. Am J Gastroenterol. 2006:101:653-63.

19. Suzuki H, Oda I, Abe S, Sekiguchi M, Nonaka S, Yoshinaga S, Saito Y, Fukagawa T, Katai $\mathrm{H}$. Clinical outcomes of early gastric cancer patients after noncurative endoscopic submucosal dissection in a large consecutive patient series. Gastric Cancer. 2017;20:679-89.

20. Yao K, Anagnostopoulos GK, Ragunath K. Magnifying endoscopy for diagnosing and delineating early gastric cancer. Endoscopy. 2009;41:462-7.

21. Rogart JN, Nagata J, Loeser CS, Roorda RD, Aslanian H, Robert ME, Zipfel WR, Nathanson MH. Multiphoton imaging can be used for microscopic examination of intact human gastrointestinal mucosa ex vivo. Clin Gastroenterol Hepatol. 2008;6:95-101.

22. Tu HH, Liu Y, Turchinovich D, Marjanovic M, Lyngso JK, Laegsgaard J, Chaney EJ, Zhao YB, You SX, Wilson WL, Xu BW, Dantus M, Boppart SA. Stain-free histopathology by programmable supercontinuum pulses. Nat Photonics. 2016;10:534-41.

23. Bremnes RM, Donnem T, Al-Saad S, Al-Shibli K, Andersen S, Sirera R, Camps C, Marinez I, Busund LT. The role of tumor stroma in cancer progression and prognosis: emphasis on carcinoma-associated fibroblasts and non-small cell lung cancer. J Thorac Oncol. 2011;6:209-17.

24. Condeelis J, Segall JE. Intravital imaging of cell movement in tumours. Nat Rev Cancer. 2003;3:921-30.

25. Provenzano PP, Eliceiri KW, Keely PJ. Multiphoton microscopy and fluorescence lifetime imaging microscopy (FLIM) to monitor metastasis and the tumor microenvironment. Clin Exp Metastasis. 2009;26:357-70.

26. Brown E, McKee T, diTomaso E, Pluen A, Seed B, Boucher $Y$, Jain RK. Dynamic imaging of collagen and its modulation in tumors in vivo using second-harmonic generation. Nat Med. 2003;9:796-800.

27. He K, Zhao L, Huang X, Ding Y, Liu L, Wang X, Wang M, Zhang Y, Fan Z. Label-free imaging for T staging of gastric carcinoma by multiphoton microscopy. Lasers Med Sci. 2018;33:871-82.

28. Sidani M, Wyckoff J, Xue C, Segall JE, Condeelis J. Probing the microenvironment of mammary tumors using multiphoton microscopy. J Mammary Gland Biol Neoplasia. 2006;11:151-63.

29. Provenzano PP, Eliceiri KW, Campbell JM, Inman DR, White JG, Keely PJ. Collagen reorganization at the tumor-stromal interface facilitates local invasion. BMC Med. 2006;4:12-26.

30. Eto $T$, Suzuki H, Honda A, Nagashima Y. The changes of the stromal elastotic framework in the growth of peripheral lung adenocarcinomas. Cancer. 1996;77:646-56.

31. Rivera DR, Brown CM, Ouzounov DG, Pavlova I, Kobat D, Webb WW, Xu C. Compact and flexible raster scanning multiphoton endoscope capable of imaging unstained tissue. Proc Nat Acad Sci U S A. 2011; 108:17598-603.

32. Huland DM, Brown CM, Howard SS, Ouzounov DG, Pavlova I, Wang K, Rivera DR, Webb WW, Xu C. In vivo imaging of unstained tissues using long gradient index lens multiphoton endoscopic systems. Biomedical Optics Express. 2012;3:1077-85.

33. Helmchen F, Denk W. Deep tissue two-photon microscopy. Nat Methods. 2005:2:932-40

34. Wang T, Li Q, Xiao P, Ahn J, Kim YE, Park Y, Kim M, Song M, Chung E, Chung WK, Ahn GO, Kim S, Kim P, Myung SJ, Kim KH. Gradient index lens based combined two-photon microscopy and optical coherence tomography. Opt Express. 2014;22:12962-70.

35. Fu L, Jain A, Cranfield C, Xie H, Gu M. Three-dimensional nonlinear optical endoscopy. J Biomed Opt. 2007;12:040501.

36. Hoy CL, Ferhanoglu O, Yildirim M, Piyawattanametha W, Ra H, Solgaard O, Ben-Yakar A. Optical design and imaging performance testing of a 9.6-mm diameter femtosecond laser microsurgery probe. Opt Express. 2011;19: 10536-52.

37. Rivera DR, Brown CM, Ouzounov DG, Webb WW, Xu C. Multifocal multiphoton endoscope. Opt Lett. 2012;37:1349-51.

38. D'Annibale A, Morpurgo E, Fiscon V, Trevisan P, Sovernigo G, Orsini C, Guidolin D. Robotic and laparoscopic surgery for treatment of colorectal diseases. Dis Colon Rectum. 2004;47:2162-8.

39. Coratti A, Fernandes E, Lombardi A, Di Marino M, Annecchiarico M, Felicioni L, Giulianotti PC. Robot-assisted surgery for gastric carcinoma: five years follow-up and beyond: A single western center experience and long-term oncological outcomes. Eur J Surg Oncol. 2015;41:1106-13.

40. Huang KH, Lan YT, Fang WL, Chen JH, Lo SS, Hsieh MC, Li AF, Chiou SH, Wu $\mathrm{CW}$. Initial experience of robotic gastrectomy and comparison with open and laparoscopic gastrectomy for gastric cancer. J Gastrointest Surg. 2012; 16:1303-10.
Ready to submit your research? Choose BMC and benefit from:

- fast, convenient online submission

- thorough peer review by experienced researchers in your field

- rapid publication on acceptance

- support for research data, including large and complex data types

- gold Open Access which fosters wider collaboration and increased citations

- maximum visibility for your research: over $100 \mathrm{M}$ website views per year

At BMC, research is always in progress.

Learn more biomedcentral.com/submissions 